\title{
LAPLACE TIME TUNING TRANSFORM IN DIGITAL SIGNAL PROCESSING
}

\author{
G. Britto Antony Xavier ${ }^{1}$, S.U. Vasantha $\operatorname{Kumar}^{2}$ and M. Meganathan ${ }^{3}$ \\ 1,2,3 Department of Mathematics, Sacred Heart College, \\ Tirupattur - 635601, Vellore District, Tamil Nadu, S.India. \\ 1 e-mail : brittoshc@gmail.com
}

\begin{abstract}
In this paper, we derive a series of new theorems and formulae on certain type of multi-series by equating closed and summation solutions of generalized difference equation with trignometric functions. We generate Discrete Laplace Transform with Time Tuning Factor $\ell$ in the field of Digital Signal Processing (DSP). Suitable examples with relevant diagrams which are generated and verified using MATLAB are inserted to validate our main results.
\end{abstract}

Key words: Generalized difference equation, Summation solution, Closed form solution, m-series, Laplace Transform and Time tuning factor.

AMS Subject classification: 39A70, 47B39, 39A10, 44A10, 65Q10, 92C55.

\section{INTRODUCTION}

Digital Signal Processing (DSP) has revolutionized many areas in science and engineering such as space, medicine, commerce, military, technology and communication. DSP is made effectively possible by Laplace Transform (LT) and Discrete Laplace Transform (DLT) which changes a signal in the time domain into frequency s-domain [15]. The Laplace transforms are practical in the view of fast decay factor $e^{-s x}$. Construction of polynomial filters for detection of peaks in periodic signals in DSP is developed in [16]. With numerical computation and MATLAB obtaining exact solutions for Dirichlet-Neumann inverse problem are discussed in [4]. In practice, many applications of Laplace Transform (LT) and Discrete Laplace Transform (DLT) are discussed by several authors $[3,9,14,10]$.

$$
\text { The LT and DLT are respectively defined as } L[f(t)]=\int_{0}^{\infty} f(t) e^{-s t} d t \quad \text { and }
$$

$L[f(n)]=\sum_{n=0}^{\infty} f(n) e^{-s n}, s>0$. From the basic difference identity $\left.\Delta^{-1} x_{n}\right|_{0} ^{\infty}=\sum_{n=0}^{\infty} x_{n}$ [1], the DLT can be expressed as $L[f(n)]=\Delta^{-1} f(n) e^{-s n}$. Let $u(k)$ be input signal(functions) and $\ell$ be the time interval between two successive signals.

In [8], authors have defined a new type Laplace Transform as

$$
L_{\ell} u(k)=\left.\ell \Delta_{\ell}^{-1} u(k) e^{-s k}\right|_{0} ^{\infty}=\ell \sum_{r=0}^{\infty} u(r \ell) e^{-s r \ell} .
$$

This transform is called as Generalized Laplace Transform (GLT) and it lies in between DLT and LT. The GLT becomes DLT and LT when $\ell=1$ and $\ell \rightarrow 0$ respectively [2,3]. If we take $\ell$ as time between two successive signals in DSP (1) becomes Laplace Time Tuning Transform (LTTT). To develop certain theories on LTTT we need to reveal the basic theory of $\Delta_{\ell}$ and their inverse [5, 12].

The main definition of fractional difference equation (as done in [13]) is the $v$ fractional sum of $f(t)$ by $\Delta^{-v} f(t)=\frac{1}{\Gamma_{(v)}} \sum_{s=a}^{t-v} \frac{\Gamma_{(t-s)}}{\Gamma_{(t-s-(v-1))}} f(s)$, where $v>0$.

When $v=m$ is a positive integer, if we replace $f(t)$ by $u(k)$ and $\Delta$ by $\Delta_{\ell}$.

(i.e. $\left.\Delta_{\ell} u(k)=u(k+\ell)-u(k), k \in[0, \infty), \ell>0\right)$, we arrive an m-series 


$$
\Delta_{\ell}^{-m} u(k)=\sum_{r=m}^{\left[\frac{k}{\ell}\right]} \frac{(r-1)^{(m-1)}}{(m-1) !} u(k-r \ell),
$$

where $(r-1)^{(m-1)}=(r-1)(r-2) \cdots(r-m+1)$ and $\left[\frac{k}{\ell}\right]$ is the integer part of $\frac{k}{\ell}$. This m-series is a numerical solution of the $\mathrm{m}^{\text {th }}$ order generalized difference equation

$$
\Delta_{\ell}^{m} v(k)=u(k), k \in[0, \infty), \ell>0 .
$$

When $m=1, k \rightarrow \infty$, replacing $u(k)$ by $\ell u(k) e^{-s k}$ and by rearranging the terms, the equation (2) for $\ell u(k) e^{-s k}$ becomes LTTT given in (1).

Let $\ell>0$ be time tuning factor of two successive signals and $u(k)$ be a real valued function on $[0, \infty)$ representing input signal of a system in DSP. In this paper, by deriving several formulas on m-series to circular functions with respect to $\ell$, we analyze the Laplace Time Tuning Transform in the field of Digital Signal Processing.

\section{PRELIMINARIES}

Before stating and proving our results, we present some notations, basic definitions and preliminary results which will be useful for further subsequent discussions. $\Delta_{\ell}^{-m} u(k) \|_{(m-1) \ell+j}^{k}=$ $\left.\Delta_{\ell}^{-1}\left(\left.\cdots \Delta_{\ell}^{-1}\left(\left.\Delta_{\ell}^{-1} u(k)\right|_{j} ^{k}\right)\right|_{\ell+j} ^{k} \cdots\right)\right|_{(m-1) \ell+j} ^{k}$, where $\left.\Delta_{\ell}^{-1} u(k)\right|_{j} ^{k}=u_{1}(k)=\Delta_{\ell}^{-1} u(k)-\Delta_{\ell}^{-1} u(j)$, $\left.\Delta_{\ell}^{-1}\left(\left.\Delta_{\ell}^{-1} u(k)\right|_{j} ^{k}\right)\right|_{\ell+j} ^{k}=u_{2}(k)=\Delta_{\ell}^{-1} u_{1}(k)-\Delta_{\ell}^{-1} u_{1}(\ell+j)$, and so on, $j=k-\left[\frac{k}{\ell}\right] \ell$, $\mathbb{N}_{\ell}(j)=\{j, \ell+j, 2 \ell+j, \cdots\}$ and $\mathbb{N}_{1}(j)=\mathbb{N}(j) . c_{j}$ is a constant for all $k \in \mathbb{N}_{\ell}(j)$ and for any positive integer $m$.

Also, $L_{m-1}=\{1,2, \ldots, m-1\}, 0\left(L_{m-1}\right)=\{\phi\}, \phi$ is empty set, $t\left(L_{m-1}\right)=$ set of all subsets of size $t$ from the set $L_{m-1}$ such that if $\left\{m_{1}, m_{2}, \cdots, m_{t}\right\} \in t\left(L_{m-1}\right)$ then $m_{1}<m_{2}<\cdots<m_{t}$ for $t=1,2, \cdots, m-1, \wp\left(L_{m-1}\right)=\bigcup_{t=0}^{m-1} t\left(L_{m-1}\right)$ is the power set of $L_{m-1}, \sum_{t=1}^{m-1} f(t)=0$ for $m \leq 1$, and $\prod_{i=2}^{t} f(i)=1$ for $t \leq 1$, and $\left\{m_{t}\right\} \in t\left(L_{m-1}\right)$ means that $\left\{m_{1}, m_{2}, \cdots, m_{t}\right\} \in t\left(L_{m-1}\right)$.

In [7] the authors have introduced generalized polynomial factorial $k_{\ell}^{(m)}=k(k-\ell)(k-2 \ell) \cdots(k-(m-1) \ell)$. Using Stirling numbers of first kind $s_{r}^{m}$ and second kind $S_{r}^{m}$, the following identities have been obtained:

$$
\text { (i) } k_{\ell}^{(m)}=\sum_{r=1}^{m} s_{r}^{m} \ell^{m-r} k^{r} \text {, (ii) } k^{m}=\sum_{r=1}^{m} S_{r}^{m} \ell^{m-r} k_{\ell}^{(r)}, \text { (iii) } \Delta_{\ell} k_{\ell}^{(m)}=(m \ell) k_{\ell}^{(m-1)} \text {. }
$$

Definition 2.1 [11] Let $u(k), k \in[0, \infty)$ be a real valued function. The generalized difference operator $\Delta_{\ell}$ on $u(k)$ is defined as;

$$
\Delta_{\ell} u(k)=u(k+\ell)-u(k), k \in[0, \infty), \ell>0,
$$

and the inverse of $\Delta_{\ell}$ on $u(k)$ is defined as,

$$
\text { if } \Delta_{\ell} v(k)=u(k) \text {, then } v(k)=\Delta_{\ell}^{-1} u(k)+c_{j} .
$$

Ingeneral, $\Delta_{\ell}^{-v}=\Delta_{\ell}^{-1}\left(\Delta_{\ell}^{-(v-1)}\right)$. 
Lemma 2.2 [7] Let $p$ and $q$ be any real numbers. If $1-\cos p \ell \neq 0$, then

$$
\Delta_{\ell}^{-1} \sin p k=\frac{\sin p(k-\ell)-\sin p k}{2(1-\cos p \ell)}+c_{j}
$$

and

$$
\Delta_{\ell}^{-1} \cos p k=\frac{\cos p(k-\ell)-\cos p k}{2(1-\cos p \ell)}+c_{j}
$$

are solutions of (3) for $u(k)=\sin p k$ and $\cos p k$ respectively when $m=1$.

Remark 2.3 Throughout this paper, we denote $P=p\left(n_{2}-2 s_{2}\right)+q\left(n_{3}-2 s_{3}\right)$ and $\bar{P}=p\left(n_{2}-2 s_{2}\right)-q\left(n_{3}-2 s_{3}\right)$. $P$ and $\bar{P}$ are depending on $n_{2}, n_{3}, s_{2}, s_{3}, p$ and $q$.

To evaluate $\Delta_{\ell}^{-1} u(k) e^{-s k}$ (LTTT) we present the following lemmas and theorem.

Lemma 2.4 [6] Let $v(k)$ and $w(k)$ be two real valued functions. Then,

$$
\Delta_{\ell}^{-1}[v(k) w(k)]=v(k) \Delta_{\ell}^{-1} w(k)-\Delta_{\ell}^{-1}\left[\Delta_{\ell}^{-1} w(k+\ell) \Delta_{\ell} v(k)\right] .
$$

Lemma 2.5 [11] Let $k \in[\ell, \infty)$. Then,

$$
\left.\Delta_{\ell}^{-1} e^{-k}\right|_{j} ^{k}=\frac{e^{-k}}{e^{-\ell}-1}-\frac{e^{-j}}{e^{-\ell}-1} .
$$

Lemma 2.6 [11] Let $n$ be any non-negative integer. Then,

$$
\left.\Delta_{\ell}^{-1} k_{\ell}^{(n)}\right|_{j} ^{k}=\frac{k_{\ell}^{(n+1)}}{\ell(n+1)}-\frac{j_{\ell}^{(n+1)}}{\ell(n+1)} .
$$

Theorem 2.7 [6] (m-series formula) Let $m \in \mathbb{N}(2)$. Then, we find

$$
\begin{gathered}
\sum_{r=m}^{\left[\frac{k}{\ell}\right]} \frac{(r-1)^{(m-1)}}{(m-1) !} u(k-r \ell)=\left.\Delta_{\ell}^{-m} u(k)\right|_{(m-1) \ell+j} ^{k}+\sum_{t=1\left\{m_{1}, \ldots, m_{t}\right\} \in t\left(L_{m-1}\right)}^{m-1}(-1)^{t} \times \\
\left.\left(\Delta_{\ell}^{-m_{1}} u\left(\left(m_{1}-1\right) \ell+j\right)\right) \frac{k_{\ell}^{\left(m-m_{t}\right)}}{\left(m-m_{t}\right) ! \ell^{m-m_{t}}} \prod_{i=2}^{t} \frac{\left(\left(m_{i}-1\right) \ell+j\right)_{\ell}^{\left(m_{i}-m_{i-1}\right)}}{\left(m_{i}-m_{i-1}\right) ! \ell^{m_{i}-m_{i-1}}}\right|_{(m-1) \ell+j} ^{k}
\end{gathered}
$$

LHS of (12) gives m-series and RHS provides the value of the m-series to $u(k)$.

\section{SUMMATION OPERATOR}

In this section, we introduce some summation notations for representing m-series in a simple manner. (i) $\mathbb{T}_{\text {oo }}=\{(1,0),(0,1)\}$, (ii) $\mathbb{T}_{\text {oe }}=\{(1,0),(0,1),(1,1)\}$,

(iii) $\mathbb{T}_{e o}=\{(1,0),(0,1),(1,-1)\}$, (iv) $\mathbb{T}_{e e}=\{(1,0),(0,1),(1,1),(1,-1)\}$,

(v) $\left(\left(n_{2}\right)\right)=\left(\begin{array}{l}n_{2} \\ \frac{n_{2}}{2}\end{array}\right)^{-u v\left(\frac{u-v}{u^{2}+v^{2}}\right)}$, (vi) $\left(\left(n_{3}\right)\right)=\left(\begin{array}{l}n_{3} \\ \frac{n_{3}}{2}\end{array}\right)^{u v\left(\frac{u+v}{u^{2}+v^{2}}\right)}$

(1) If $n_{2}$ and $n_{3}$ are odd positive integers, then

$$
\sum_{\left(n_{2}, n_{3}\right)}^{s, c}=\frac{(-1)^{\frac{n_{2}-1}{2}}}{2^{n_{2}+n_{3}-1}} \sum_{s_{2}=0}^{\frac{n_{2}-1}{2}} \sum_{s_{3}=0}^{\frac{n_{3}-1}{2}}(-1)^{s_{2}} \frac{n_{2}^{\left(s_{2}\right)}}{s_{2} !} \frac{n_{3}^{\left(s_{3}\right)}}{s_{3} !}
$$

(2) If $n_{2}$ and $n_{3}$ are even positive integers, then 


$$
\sum_{\left[n_{2}, n_{3}\right]}^{s, c}=\frac{(-1)^{\frac{n_{2}}{2}}}{2^{n_{2}+n_{3}-1}} \sum_{s_{2}=0}^{\frac{n_{2}-2}{2}} \sum_{s_{3}=0}^{\frac{n_{3}-2}{2}}(-1)^{s_{2}} \frac{n_{2}^{\left(s_{2}\right)}}{s_{2} !} \frac{n_{3}^{\left(s_{3}\right)}}{s_{3} !}
$$

Operators used for product of circular, k-factorial and exponential functions.

$$
\sum_{n_{1}\left(n_{2}, n_{3}\right)}^{s, c, m+s_{1}}=\frac{(-1)^{\frac{n_{2}-1}{2}}}{2^{n_{2}+n_{3}-1}} \sum_{s_{1}=0}^{n_{1}} \sum_{s_{2}=0}^{\frac{n_{2}-1}{2}} \sum_{s_{3}=0}^{2} \sum_{s_{4}=0}^{\frac{m+s_{1}}{2}} \frac{n_{1}^{\left(s_{1}\right)} n_{2}^{\left(s_{2}\right)} n_{3}^{\left(s_{3}\right)}\left(m+s_{1}\right)^{\left(s_{4}\right)}}{(-1)^{s_{1}+s_{2}+s_{4}} s_{1} ! s_{2} ! s_{3} ! s_{4} !}
$$

Operators used for product of circular functions with exponential alone.

$$
\sum_{\left(n_{2}, n_{3}\right) m}^{s, c, m}=\frac{(-1)^{\frac{n_{2}-1}{2}}}{2^{n_{2}+n_{3}-2}} \sum_{s_{1}=0 s_{2}=0}^{m} \sum_{s_{3}=0}^{\frac{n_{2}-1}{2}} \sum^{\frac{n_{3}-1}{2}}(-1)^{s_{1}+s_{2}} \frac{m^{\left(s_{1}\right)}}{s_{1} !} \frac{n_{2}^{\left(s_{2}\right)}}{s_{2} !} \frac{n_{3}^{\left(s_{3}\right)}}{s_{3} !}
$$

\section{MAIN RESULTS}

In this section we assume that $P \ell_{i}, \bar{P} \ell_{i},\left(\frac{P+\bar{P}}{2}\right) \ell_{i},\left(\frac{P-\bar{P}}{2}\right) \ell_{i}$ are not multiple of $2 \pi$ for $i=1,2, \cdots, n, m_{(r)}=m(m+1)(m+2) \cdots(m+(r-1))$ and $s, p$ and $q$ are any real numbers. We find $\mathrm{m}$-series of product of polynomial factorial, sine and cosine functions.

\subsection{Inverse operator on product of two functions}

Theorem 4.1 If $n_{2}$ and $n_{3}$ are odd positive integers, then we have $\Delta_{\ell}^{-m}\left(k_{\ell}^{\left(n_{1}\right)} \sin ^{n_{2}} p k \cos ^{n_{3}} q k\right)$

$$
=\sum_{n_{1}\left(n_{2}, n_{3}\right)}^{s, c, m+s_{1}} \sum_{(u, v) \in \mathbb{T}_{o o}} m_{\left(s_{1}\right)} \ell^{s_{1}} k_{\ell}^{\left(n_{1}-s_{1}\right)} \frac{\sin \left(\frac{u P+v \bar{P}}{u^{2}+v^{2}}\right)\left(k-\left(m-s_{4}\right) \ell\right)}{\left(2\left(1-\cos \left(\frac{u P+v \bar{P}}{u^{2}+v^{2}}\right) \ell\right)\right)^{m+s_{1}}} .
$$

Proof. Take $f_{1}(k)=k_{\ell}^{(1)} \sin ^{n_{2}} p k_{\cos }{ }^{n_{3}} q k$. Using (7), (8), (9) and changing the powers of sin and cos into linear, we find that

$$
\Delta_{\ell}^{-1} f_{1}(k)=\sum_{1\left(n_{2}, n_{3}\right)}^{s, c, 1+s_{1}} \sum_{(u, v) \in \mathbb{T}_{o o}} 1_{\left(s_{1}\right)} \ell^{s_{1}} k_{\ell}^{\left(1-s_{1}\right)} \frac{\sin \left(\frac{u P+v \bar{P}}{u^{2}+v^{2}}\right)\left(k-\left(1-s_{4}\right) \ell\right)}{\left(2\left(1-\cos \left(\frac{u P+v \bar{P}}{u^{2}+v^{2}}\right) \ell\right)\right)^{1+s_{1}}} .
$$

Applying $\Delta_{\ell}^{-1}$ on both sides to above equation, we get

$$
\Delta_{\ell}^{-2} f_{1}(k)=\sum_{1\left(n_{2}, n_{3}\right)(u, v) \in \mathbb{T}_{o o}}^{s, c, 2+s_{1}} 2_{\left(s_{1}\right)} \ell^{s_{1}} k_{\ell}^{\left(1-s_{1}\right)} \frac{\sin \left(\frac{u P+v \bar{P}}{u^{2}+v^{2}}\right)\left(k-\left(2-s_{4}\right) \ell\right)}{\left(2\left(1-\cos \left(\frac{u P+v \bar{P}}{u^{2}+v^{2}}\right) \ell\right)\right)^{2+s_{1}}} .
$$

Proceeding like this, we arrive

$$
\Delta_{\ell}^{-m} f_{1}(k)=\sum_{1\left(n_{2}, n_{3}\right)}^{s, c, m+s_{1}} \sum_{(u, v) \in \mathbb{T}_{o o}} m_{\left(s_{1}\right)} \ell^{s_{1}} k_{\ell}^{\left(1-s_{1}\right)} \frac{\sin \left(\frac{u P+v \bar{P}}{u^{2}+v^{2}}\right)\left(k-\left(m-s_{4}\right) \ell\right)}{\left(2\left(1-\cos \left(\frac{u P+v \bar{P}}{u^{2}+v^{2}}\right) \ell\right)\right)^{m+s_{1}}} .
$$


Similarly, if we take $f_{2}(k)=k_{\ell}^{(2)} \sin ^{n_{2}} p k_{\cos ^{3}} q k$, we find

$$
\Delta_{\ell}^{-m} f_{2}(k)=\sum_{2\left(n_{2}, n_{3}\right)}^{s, c, m+s_{1}} \sum_{(u, v) \in \mathbb{T}_{o o}} m_{\left(s_{1}\right)} \ell^{s_{1}} k_{\ell}^{\left(2-s_{1}\right)} \frac{\sin \left(\frac{u P+v \bar{P}}{u^{2}+v^{2}}\right)\left(k-\left(m-s_{4}\right) \ell\right)}{\left(2\left(1-\cos \left(\frac{u P+v \bar{P}}{u^{2}+v^{2}}\right) \ell\right)\right)^{m+s_{1}}} .
$$

Continuing this process (m-inverse) upto $n_{1}$, we get $\Delta_{\ell}^{-m} f_{n_{1}}(k)$ given in (13).

Theorem 4.2 If $n_{2}$ and $n_{3}$ are even positive integers, then

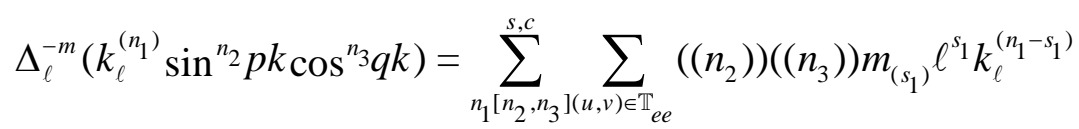

$$
\begin{aligned}
& \times\left\{\frac{\cos \left(\frac{u P+v \bar{P}}{u^{2}+v^{2}}\right)\left(k-\left(m-s_{4}\right) \ell\right)}{\left(2\left(1-\cos \left(\frac{u P+v \bar{P}}{u^{2}+v^{2}}\right) \ell\right)\right)^{m+s_{1}}}+n_{1} ! \frac{n_{2}^{\left(\frac{n_{2}}{2}\right)}}{\frac{n_{2}}{2} !} \frac{n_{3}^{\left(\frac{n_{3}}{2}\right)}}{\frac{n_{3}}{2} !} \frac{k_{\ell}^{\left(m+n_{1}\right)}}{\left(m+n_{1}\right) ! \ell^{m+n_{1}}}\right\} .
\end{aligned}
$$

Proof. The proof is similar to the proof of Theorem 4.1.

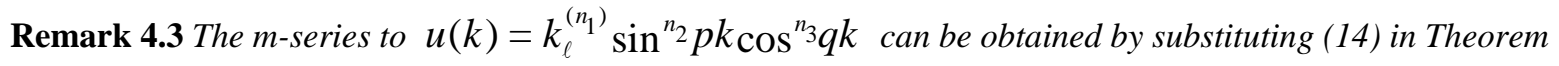
2.7.

Theorem 4.4 If $n_{2}$ and $n_{3}$ are odd positive integers, then we have $\Delta_{\ell}^{-m}\left(e^{-s k} \sin ^{n_{2}} p k_{\cos }{ }^{n_{3}} q k\right)$

$$
=\sum_{\left(n_{2}, n_{3}\right)(u, v) \in \mathbb{T}_{o o}}^{s, c, m} e^{-s k} e^{s_{1} s \ell} \frac{\sin \left(\frac{u P+v \bar{P}}{u^{2}+v^{2}}\right)\left(k-\left(m-s_{1}\right) \ell\right)}{\left(2\left(\cosh s \ell-\cos \left(\frac{u P+v \bar{P}}{u^{2}+v^{2}}\right) \ell\right)\right)^{m}} .
$$

Proof. After changing the powers of $\sin$ and cos into linear, we get

$$
\begin{aligned}
& \Delta_{\ell}^{-1}\left(e^{-s k} \sin ^{n_{2}} p k \cos ^{n_{3}} q k\right)=\sum_{\left(n_{2}, n_{3}\right)}^{s, c} \Delta_{\ell}^{-1}\left(e^{-s k}(\sin P k+\sin \bar{P} k)\right)=\operatorname{Impartof} \sum_{\left(n_{2}, n_{3}\right)}^{s, c} \\
& \times \Delta_{\ell}^{-1}\left(e^{-s k}\left(e^{i P k}+e^{i \bar{P} k}\right)\right)=\operatorname{Impartof} \sum_{\left(n_{2}, n_{3}\right)}^{s, c}\left(\frac{e^{(i P-s) k}}{e^{(i P-s) \ell}-1}+\frac{e^{(i \bar{P}-s) k}}{e^{(i \bar{P}-s) \ell}-1}\right),
\end{aligned}
$$

After simplification, we get $\Delta_{\ell}^{-1}\left(e^{-s k} \sin ^{n_{2}} p k \cos ^{n_{3}} q k\right)$

$$
=\sum_{\left(n_{2}, n_{3}\right)(u, v) \in \mathbb{T}_{o o}}^{s, c, 1} e^{-s k} e^{s_{1} s \ell} \frac{\sin \left(\frac{u P+v \bar{P}}{u^{2}+v^{2}}\right)\left(k-\left(1-s_{1}\right) \ell\right)}{\left(2\left(\cosh s \ell-\cos \left(\frac{u P+v \bar{P}}{u^{2}+v^{2}}\right) \ell\right)\right)}
$$

Applying $\Delta_{\ell}^{-1}$ on both sides to equation (16), we get

$$
\Delta_{\ell}^{-2}\left(e^{-s k} \sin ^{n_{2}} p k_{\cos }{ }^{n_{3}} q k\right)
$$

$$
=\sum_{\left(n_{2}, n_{3}\right)(u, v) \in \mathbb{T}_{o o}, c, 2} e^{-s k} e^{s_{1} s \ell} \frac{\sin \left(\frac{u P+v \bar{P}}{u^{2}+v^{2}}\right)\left(k-\left(2-s_{1}\right) \ell\right)}{\left(2\left(\cosh s \ell-\cos \left(\frac{u P+v \bar{P}}{u^{2}+v^{2}}\right) \ell\right)\right)^{2}}
$$

Continuing this process upto m-inverse, we get (15). 
Remark 4.5 The m-series to $u(k)=e^{-s k} \sin ^{n_{2}} p k \cos ^{n_{3}} q k$ can be obtained by substituting (15) in Theorem 2.7.

\subsection{Inverse operator on product of three functions}

Theorem 4.6 If $n_{2}$ and $n_{3}$ are odd positive integers, then

$$
\Delta_{\ell}^{-m}\left(k_{\ell}^{\left(n_{1}\right)} e^{-s k} \sin ^{n_{2}} p k_{\left.\cos ^{n_{3}} q k\right)}\right.
$$

$$
=\sum_{n_{1}\left(n_{2}, n_{3}\right)(u, v) \in \mathbb{T}_{o o}}^{s, c, m+s_{1}} \frac{k_{\ell}^{\left(n_{1}-s_{1}\right)} m_{\left(s_{1}\right)}}{\ell^{-s_{1}} e^{s\left(k+s_{1} \ell\right)}} \frac{e^{s_{4} s \ell} \sin \left(\frac{u P+v \bar{P}}{u^{2}+v^{2}}\right)\left(k-\left(m-s_{4}\right) \ell\right)}{\left(2\left(\cosh s \ell-2 \cos \left(\frac{u P+v \bar{P}}{u^{2}+v^{2}}\right) \ell\right)\right)^{m+s_{1}}} .
$$

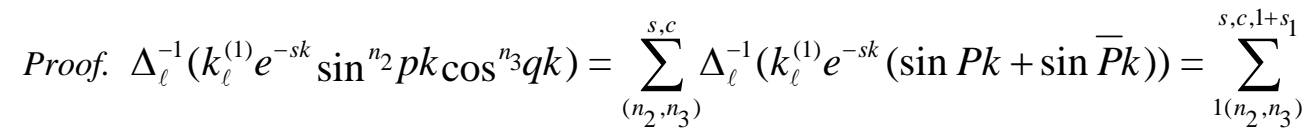

$$
\times \sum_{(u, v) \in \mathbb{T}_{o o}} 1_{\left(s_{1}\right)} \ell^{s_{1}} k_{\ell}^{\left(1-s_{1}\right)} e^{-s\left(k+s_{1} \ell\right)} e^{s_{4}{ }^{s \ell}} \frac{\sin \left(\frac{u P+v \bar{P}}{u^{2}+v^{2}}\right)\left(k-\left(1-s_{4}\right) \ell\right)}{\left(2\left(\cosh s \ell-\cos \left(\frac{u P+v \bar{P}}{u^{2}+v^{2}}\right) \ell\right)\right)^{1+s_{1}}}
$$

Applying $\Delta_{\ell}^{-1}$ on both sides, we get $\Delta_{\ell}^{-2}\left(k_{\ell}^{(1)} e^{-s k} \sin ^{n_{2}} p k_{\left.\cos ^{n_{3}} q k\right)}=\sum_{1\left(n_{2}, n_{3}\right)}^{s, c, 2+s_{1}}\right.$

$$
\times \sum_{(u, v) \in \mathbb{T}_{o o}} 2_{\left(s_{1}\right)} \ell^{s_{1}} k_{\ell}^{\left(1-s_{1}\right)} e^{-s\left(k+s_{1} \ell\right)} e^{s_{4}{ }^{s \ell}} \frac{\sin \left(\frac{u P+v \bar{P}}{u^{2}+v^{2}}\right)\left(k-\left(2-s_{4}\right) \ell\right)}{\left(2\left(\cosh s \ell-\cos \left(\frac{u P+v \bar{P}}{u^{2}+v^{2}}\right) \ell\right)\right)^{2+s_{1}}}
$$

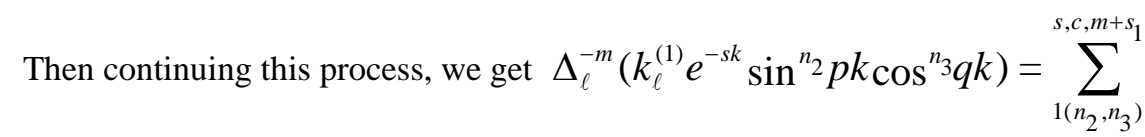

$$
\times \sum_{(u, v) \in \mathbb{T}_{o o}} m_{\left(s_{1}\right)} \ell^{s_{1}} k_{\ell}^{\left(1-s_{1}\right)} e^{-s\left(k+s_{1} \ell\right)} e^{s_{4}{ }^{s \ell}} \frac{\sin \left(\frac{u P+v \bar{P}}{u^{2}+v^{2}}\right)\left(k-\left(m-s_{4}\right) \ell\right)}{\left(2\left(\cosh s \ell-\cos \left(\frac{u P+v \bar{P}}{u^{2}+v^{2}}\right) \ell\right)\right)^{m+s_{1}}}
$$

Similarly we can obtain, $\Delta_{\ell}^{-m}\left(k_{\ell}^{(2)} e^{-s k} \sin ^{n_{2}} p k_{\left.\cos ^{n_{3}} q k\right)}=\sum_{2\left(n_{2}, n_{3}\right)}^{s, c, m+s_{1}}\right.$

$$
\times \sum_{(u, v) \in \mathbb{T}_{o o}} m_{\left(s_{1}\right)} \ell^{s_{1}} k_{\ell}^{\left(2-s_{1}\right)} e^{-s\left(k+s_{1} \ell\right)} e^{s_{4}{ }^{s \ell}} \frac{\sin \left(\frac{u P+v \bar{P}}{u^{2}+v^{2}}\right)\left(k-\left(m-s_{4}\right) \ell\right)}{\left(2\left(\cosh s \ell-\cos \left(\frac{u P+v \bar{P}}{u^{2}+v^{2}}\right) \ell\right)\right)^{m+s_{1}}} .
$$

Continuing this process upto $m$-inverse for $n_{1}$, we get equation (17).

Theorem 4.7 If $n_{2}$ is an odd and $n_{3}$ is an even positive integers, then

$$
\Delta_{\ell}^{-m}\left(k_{\ell}^{\left(n_{1}\right)} e^{-s k} \sin ^{n_{2}} p k \cos ^{n_{3}} q k\right)=\sum_{n_{1}\left(n_{2}, n_{3}\right](u, v) \in \mathbb{T}_{o e}}^{s, c, m+s_{1}}\left(\left(n_{2}\right)\right)\left(\left(n_{3}\right)\right) m_{\left(s_{1}\right)}
$$




$$
\times \frac{k_{\ell}^{\left(n_{1}-s_{1}\right)} e^{s_{4} s \ell}}{\ell^{-s_{1}} e^{s\left(k+s_{1} \ell\right)}} \frac{\sin \left(\frac{u P+v \bar{P}}{u^{2}+v^{2}}\right)\left(k-\left(m-s_{4}\right) \ell\right)}{\left(2\left(\cosh s \ell-\cos \left(\frac{u P+v \bar{P}}{u^{2}+v^{2}}\right) \ell\right)\right)^{m+s_{1}}} .
$$

Similarly, we can obtain equation for $n_{2}$ even and $n_{3}$ odd positive integers.

Theorem 4.8 If $n_{2}$ and $n_{3}$ are even positive integers, then

$$
\begin{aligned}
\Delta_{\ell}^{-m}\left(k_{\ell}^{\left(n_{1}\right)} e^{-s k}\right. & \sin ^{n_{2}} p k_{\left.\cos ^{n_{3}} q k\right)}=\sum_{n_{1}\left[n_{2}, n_{3}\right](u, v) \in \mathbb{T}_{e e}}^{s, c, m+s_{1}}\left(\left(n_{2}\right)\right)\left(\left(n_{3}\right)\right) \frac{k_{\ell}^{\left(n_{1}-s_{1}\right)} m_{\left(s_{1}\right)}}{\ell^{-s_{1}} e^{s\left(k+s_{1} \ell\right)}} \\
\times & \left(\frac{e^{s_{4}{ }^{s \ell}} \cos \left(\frac{u P+v \bar{P}}{u^{2}+v^{2}}\right)\left(k-\left(m-s_{4}\right) \ell\right)}{\left(2 \cosh s \ell-2 \cos \left(\frac{u P+v \bar{P}}{u^{2}+v^{2}}\right) \ell\right)^{m+s_{1}}}+\frac{n_{2}^{\left(\frac{n_{2}}{2}\right)}}{\frac{n_{2}}{2} !} \frac{n_{3}^{\left(\frac{n_{3}}{2}\right)}}{\frac{n_{3}}{2} !} \frac{1}{2\left(e^{-s \ell}-1\right)^{m+s_{1}}}\right) .
\end{aligned}
$$

Corollary 4.9 If $n_{2}$ and $n_{3}$ are odd positive integers, then

$$
\begin{aligned}
& \Delta_{\ell}^{-m}\left(k^{n_{1}} e^{-s k} \sin ^{n_{2}} p k_{\left.\cos ^{n_{3}} q k\right)}\right. \\
& =\sum_{r_{1}=1}^{n_{1}} \sum_{r_{1}\left(n_{2}, n_{3}\right)}^{s, c, m+s_{1}} \sum_{(u, v) \in \mathbb{T}_{o o}} \frac{S_{r_{1}}^{n_{1}} k_{\ell}^{\left(r_{1}-s_{1}\right)} m_{\left(s_{1}\right)}}{\ell_{1}^{r_{1}-n_{1}} \ell^{-s_{1}} e^{s\left(k+s_{1} \ell\right)}} \frac{e^{s_{4}{ }^{s \ell}} \sin (U V)\left(k-\left(m-s_{4}\right) \ell\right)}{(2 \cosh s \ell-2 \cos (U V) \ell)^{m+s_{1}}} .
\end{aligned}
$$

Proof. The proof follows by applying (ii) of (4) in equation 17 .

Remark 4.10 Hereafter we denote $\Pi(t)=\prod_{i=2}^{t} \frac{\left(\left(m_{i}-1\right) \ell+j\right)_{\ell}^{\left(m_{i}-m_{i-1}\right)}}{\left(m_{i}-m_{i-1}\right) ! \ell^{m_{i}-m_{i-1}}}$.

Theorem 4.11 If $n_{1}$ and $n_{2}$ are odd positive integers, then the m-series to (17) is

$$
\begin{aligned}
& \sum_{r=m}^{\left[\frac{k}{\ell}\right]} \frac{(r-1)^{(m-1)}}{(m-1) !}(k-r \ell)_{\ell}^{\left(n_{1}\right)} e^{-s(k-r \ell)} \sin ^{n_{2}} p(k-r \ell) \cos ^{n_{3}} q(k-r \ell)=\sum_{n_{1}\left(n_{2}, n_{3}\right)}^{s, c, m+s_{1}} \\
& \left.\sum_{(u, v) \in \mathbb{T}_{o o}} \frac{m_{\left(s_{1}\right)} k_{\ell}^{\left(n_{1}-s_{1}\right)}}{\ell^{-s_{1}} e^{s\left(k+s_{1} \ell\right)}} e^{s_{4} s \ell} \frac{\sin \left(\frac{u P+v \bar{P}}{u^{2}+v^{2}}\right)\left(k-\left(m-s_{4}\right) \ell\right)}{\left(2\left(\cosh s \ell-\cos \left(\frac{u P+v \bar{P}}{u^{2}+v^{2}}\right) \ell\right)\right)^{m+s_{1}}}\right|_{(m-1) \ell+j} ^{k}+ \\
& \sum_{t=1}^{m-1} \sum_{\left\{m_{t}\right\} \in t\left(L_{m-1}\right)} \sum_{n_{1}\left(n_{2}, n_{3}\right)(u, v) \in \mathbb{T}_{o o}}^{s, c, m_{1}+s_{1}}(-1)^{t}\left(m_{1}\right)_{\left(s_{1}\right)} \frac{\left(\left(m_{1}-1\right) \ell+j\right)_{\ell}^{\left(n_{1}-s_{1}\right)} \ell^{s_{1}} e^{s_{4} s \ell}}{e^{\left.s\left(\left(m_{1}-1\right) \ell+j\right)+s_{1} \ell\right)}} \\
& \times\left.\frac{\sin \left(\frac{u P+v \bar{P}}{u^{2}+v^{2}}\right)\left(\left(s_{4}-1\right) \ell+j\right)}{\left(2\left(\cosh s \ell-\cos \left(\frac{u P+v \bar{P}}{u^{2}+v^{2}}\right) \ell\right)\right)^{m_{1}+s_{1}}} \frac{\Pi(t) k_{\ell}^{\left(m-m_{t}\right)}}{\left(m-m_{t}\right) ! \ell^{m-m_{t}}}\right|_{(m-1) \ell+j} ^{k} .
\end{aligned}
$$

Proof. The proof is obtained by substituting (17) in Theorem (2.7) .

Remark 4.12 when $n_{3}=0$ in (21) we will get $\Delta_{\ell}^{-m}\left[k_{\ell}^{\left(n_{1}\right)} e^{-s k} \sin ^{n_{2}} p k\right]$ and when $n_{2}=0$ in (21) we will get $\Delta_{\ell}^{-m}\left[k_{\ell}^{\left(n_{1}\right)} e^{-s k} \cos ^{n_{3}} p k\right]$.

The following example illustrates a 2-series to $k_{\ell}^{(3)} e^{-2 k} \sin ^{4} 5 k \cos ^{4} 3 k$. 
Example 4.13 Consider the case $m=2, p=6, q=5, s=2, n_{1}=3, n_{2}=4$ and $n_{3}=4$, let $P=\left(6\left(3-2 r_{1}\right)+5\left(3-2 r_{2}\right)\right)$ and $\bar{P}=\left(6\left(3-2 r_{1}\right)-5\left(3-2 r_{2}\right)\right)$ In this case,

$L_{2}=\{1,2\}, 1\left(L_{2}\right)=\{\{1\},\{2\}\}$, and

$$
\text { LHS }=\sum_{r=2}^{\left[\frac{k}{\ell}\right]} \frac{(r-1)^{(2-1)}}{(2-1) !}(k-r \ell)_{\ell}^{(3)} e^{-s(k-r \ell)} \sin ^{4} p(k-r \ell) \cos ^{4} q(k-r \ell)
$$

RHS is the sum of the terms, (i) $\left.\Delta_{\ell}^{-2}\left(k_{\ell}^{(3)} e^{-2 k} \sin ^{4} p k \cos ^{4} q k\right)\right|_{\ell+j} ^{k}$ and

(ii) $\Delta_{\ell}^{-1}\left(j_{\ell}^{(3)} e^{-2 j} \sin ^{4} p j \cos ^{4} q j\right) \times\left.\frac{k_{\ell}^{(1)}}{\ell}\right|_{\ell+j} ^{k}$, where

$$
\begin{aligned}
\left.\Delta_{\ell}^{-2}\left(k_{\ell}^{(3)} e^{-s k} \sin ^{n_{2}} p k_{\cos ^{n}} q k\right)=\sum_{3\left[n_{2}, n_{3}\right](u, v) \in \mathbb{T}_{e e}}^{s, c, 2+s_{1}}\left(n_{2}\right)\right)\left(\left(n_{3}\right)\right) \frac{k_{\ell}^{\left(3-s_{1}\right)} 2_{\left(s_{1}\right)}}{e^{s\left(k+s_{1} \ell\right)} \ell^{-s_{1}}} \\
\times\left(\frac{e^{s_{4} s \ell} \cos \left(\frac{u P+v \bar{P}}{u^{2}+v^{2}}\right)\left(k-\left(2-s_{4}\right) \ell\right)}{\left(2\left(\cosh s \ell-\cos \left(\frac{u P+v \bar{P}}{u^{2}+v^{2}}\right) \ell\right)\right)^{2+s_{1}}}+\frac{n_{2}^{\left(\frac{n_{2}}{2}\right)}}{\frac{n_{2}}{2} !} \frac{n_{3}^{\left(\frac{n_{3}}{2}\right)}}{\frac{n_{3}}{2} !} \frac{1}{2\left(e^{-s \ell}-1\right)^{2+s_{1}}}\right) .
\end{aligned}
$$

\section{LAPLACE TUNING TRANSFORM AND ITS APPLICATIONS}

In this section we derive expression for Laplace Tuning Transform for the functions (input signals) sine, cosine and polynomial factorials, and discuss its applications in DSP.

Theorem 5.1 If $n_{2}$ and $n_{3}$ are odd positive integers, then $(i) L_{\ell}\left(\sin ^{n_{2}} p k \cos ^{n_{3}} q k\right)$

$$
=\ell \sum_{r=1}^{\infty}\left(e^{-s r \ell} \sin ^{n_{2}} p r \ell \cos ^{n_{3}} q r \ell\right)=\sum_{\left(n_{2}, n_{3}\right)(u, v) \in \mathbb{T}_{o o}}^{s, c, 1} \frac{-\ell \sin \left(\frac{u P+v \bar{P}}{u^{2}+v^{2}}\right)\left(s_{1}-1\right) \ell}{2\left(\cosh s \ell-\cos \left(\frac{u P+v \bar{P}}{u^{2}+v^{2}}\right) \ell\right)}
$$

(ii) ${ }_{k} L_{\ell}\left(\sin ^{n_{2}} p k \cos ^{n_{3}} q k\right)=-\ell \times \sum_{r=0}^{\infty}\left(e^{-s(k+r \ell)} \sin ^{n_{2}} p(k+r \ell) \cos ^{n_{3}} q(k+r \ell)\right)$

$$
=-\ell \times \sum_{\left(n_{2}, n_{3}\right)(u, v) \in \mathbb{T}_{o o}}^{s, c, 1} e^{-s k} e^{s_{1} s \ell} \frac{\sin \left(\frac{u P+v \bar{P}}{u^{2}+v^{2}}\right)\left(k-\left(1-s_{1}\right) \ell\right)}{2\left(\cosh s \ell-\cos \left(\frac{u P+v \bar{P}}{u^{2}+v^{2}}\right) \ell\right)}
$$

Proof. The proof of (i) follows by taking $m=1$ in (15), multiplying by $\ell$ and applying limits for $k$ from 0 to $\infty$.

Example 5.2 Taking $n_{2}=1, n_{3}=1, p=2$ and $q=3$ in Theorem (5.1), we obtain

$$
L_{\ell}(\sin 2 k \cos 3 k)=\frac{\ell^{2} \sin 5 \ell}{2(\cosh s \ell-\cos 5 \ell)}-\frac{\ell^{2} \sin \ell}{2(\cosh s \ell-\cos \ell)}=\ell \sum_{r=0}^{\infty} e^{-s r \ell} \sin 2 r \ell \cos 3 r \ell
$$

which is verified for $\ell=0.5$ and $s=5$ by MATLAB coding given below : syms $r$

$\operatorname{symsum}(0.5 * \exp (-5 * 0.5 * r) * \sin (2 * 0.5 * r) * \cos (3 * 0.5 * r), r, 0$, inf $)$

$=(0.25 * \sin (5 * 0.5)) . /(2 *(\cosh (5 * 0.5)-\cos (5 * 0.5)))-(0.25 * \sin (0.5)) . /(2 *(\cosh (5 * 0.5)-\cos (0.5)))$ 

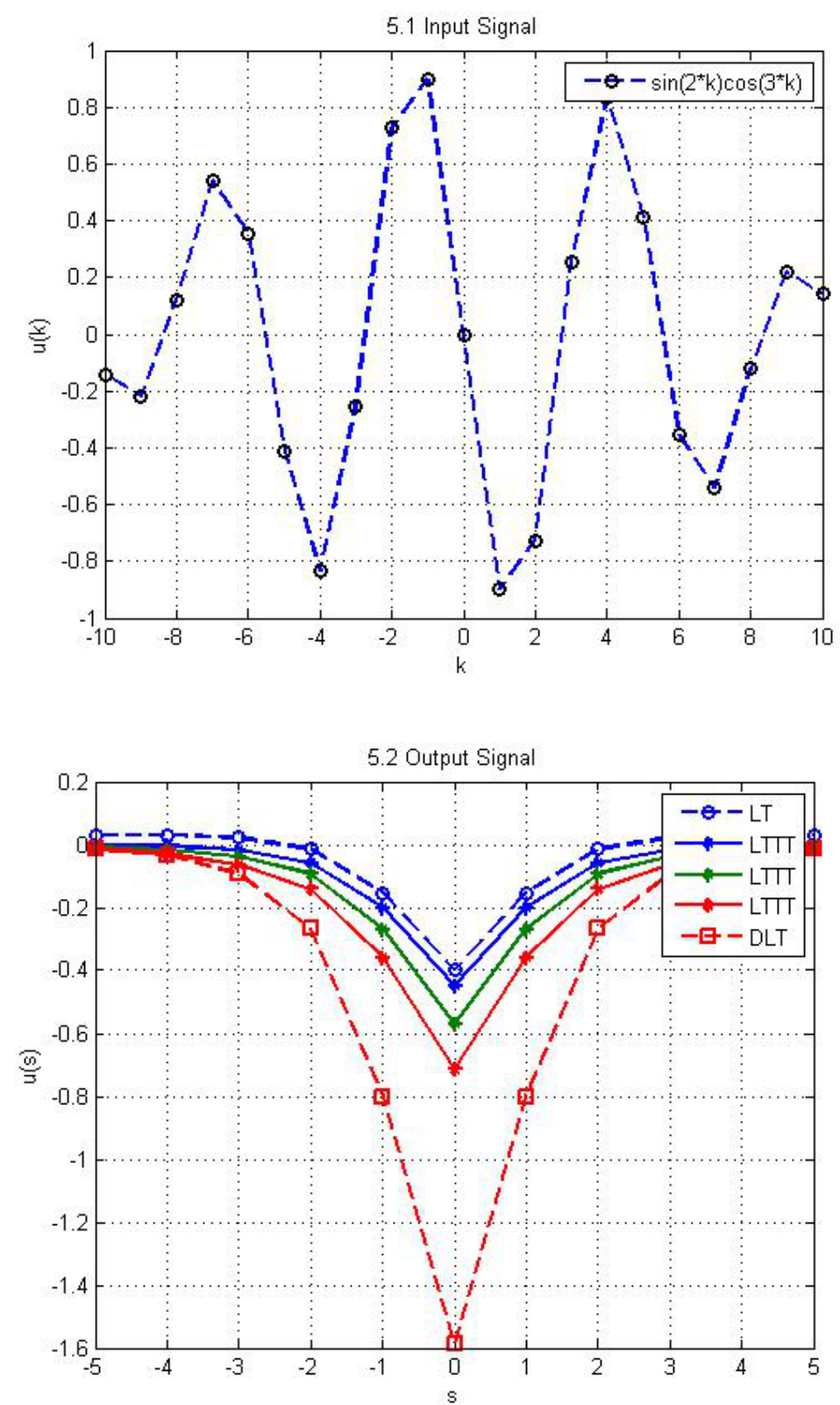

Theorem 5.3 If $n_{2}$ and $n_{3}$ are odd positive integers, then

(i) $L_{\ell}\left(k^{n_{1}} \sin ^{n_{2}} p k \cos ^{n_{3}} q k\right)=-\ell \sum_{r=0}^{\infty}(r \ell)^{n_{1}} e^{-s r \ell} \sin ^{n_{2}} p r \ell \cos ^{n_{3}} q r \ell$

$$
=\sum_{r_{1}=1}^{n_{1}} \sum_{r_{1}\left(n_{2}, n_{3}\right)}^{s, c, 1+r_{1}} \sum_{(u, v) \in \mathbb{T}_{o o}} \frac{S_{r_{1}}^{n_{1}} \ell^{n_{1}-r_{1}} 1_{\left(r_{1}\right)}}{\ell^{-r_{1}} e^{s r_{1} \ell}} \frac{e^{s_{4} s \ell} \sin (U V)\left(s_{4}-1\right) \ell}{(2 \cosh s \ell-2 \cos (U V) \ell)^{1+r_{1}}} .
$$

(ii) ${ }_{k} L_{\ell}\left(k^{n_{1}} \sin ^{n_{2}} p k_{\cos }{ }^{n_{3}} q k\right)=-\ell \times \sum_{r=0}^{\infty}(k+r \ell)^{n_{1}} e^{-s(k+r \ell)} \sin ^{n_{2}} p(k+r \ell) \cos ^{n_{3}} q(k+r \ell)$

$$
=\sum_{r_{1}=1 n_{1}\left(n_{2}, n_{3}\right)(u, v) \in \mathbb{T}_{o o}}^{n_{1}} \sum^{s, c, 1+s_{1}} \frac{S_{r_{1}}^{n_{1}} k_{\ell}^{\left(r_{1}-s_{1}\right)} 1_{\left(s_{1}\right)} \ell^{-s_{1}} e^{s\left(k+s_{1} \ell\right)}}{e^{r_{1}}} \frac{e^{s_{4} s \ell} \sin (U V)\left(k-\left(1-s_{4}\right) \ell\right)}{(2 \cosh s \ell-2 \cos (U V) \ell)^{1+s_{1}}}
$$

Proof. The proof of (i) follows by taking $m=1$ in (20), multiplying by $\ell$ and applying limits for $k$ from 0 to $\infty$. 
Example 5.4 Taking $n_{1}=2, n_{2}=1, n_{3}=1, p=5$ and $q=7$ in Theorem (5.3), we obtain $L_{\ell}\left(k^{2} \sin 5 k \cos 7 k\right)=(-\ell) \sum_{r=0}^{\infty}(r \ell)^{2} e^{-s r \ell} \sin 5 r \ell \cos 7 r \ell=\sum_{r_{1}=1}^{2} \sum_{3(1,1)}^{s, c, 1+r_{1}} \sum_{(u, v) \in \mathbb{T}_{o o}} \frac{S_{r_{1}}^{2} \ell^{2-r_{1}} 1_{\left(r_{1}\right)}}{\ell^{-r_{1}} e^{s r_{1} \ell}} \frac{e^{s_{4} s \ell} \sin (U V)\left(s_{4}-1\right) \ell}{(2 \cosh s \ell-2 \cos (U V) \ell)^{1+r_{1}}}$ which is verified for $\ell=0.5$ and $s=10$ by MATLAB.
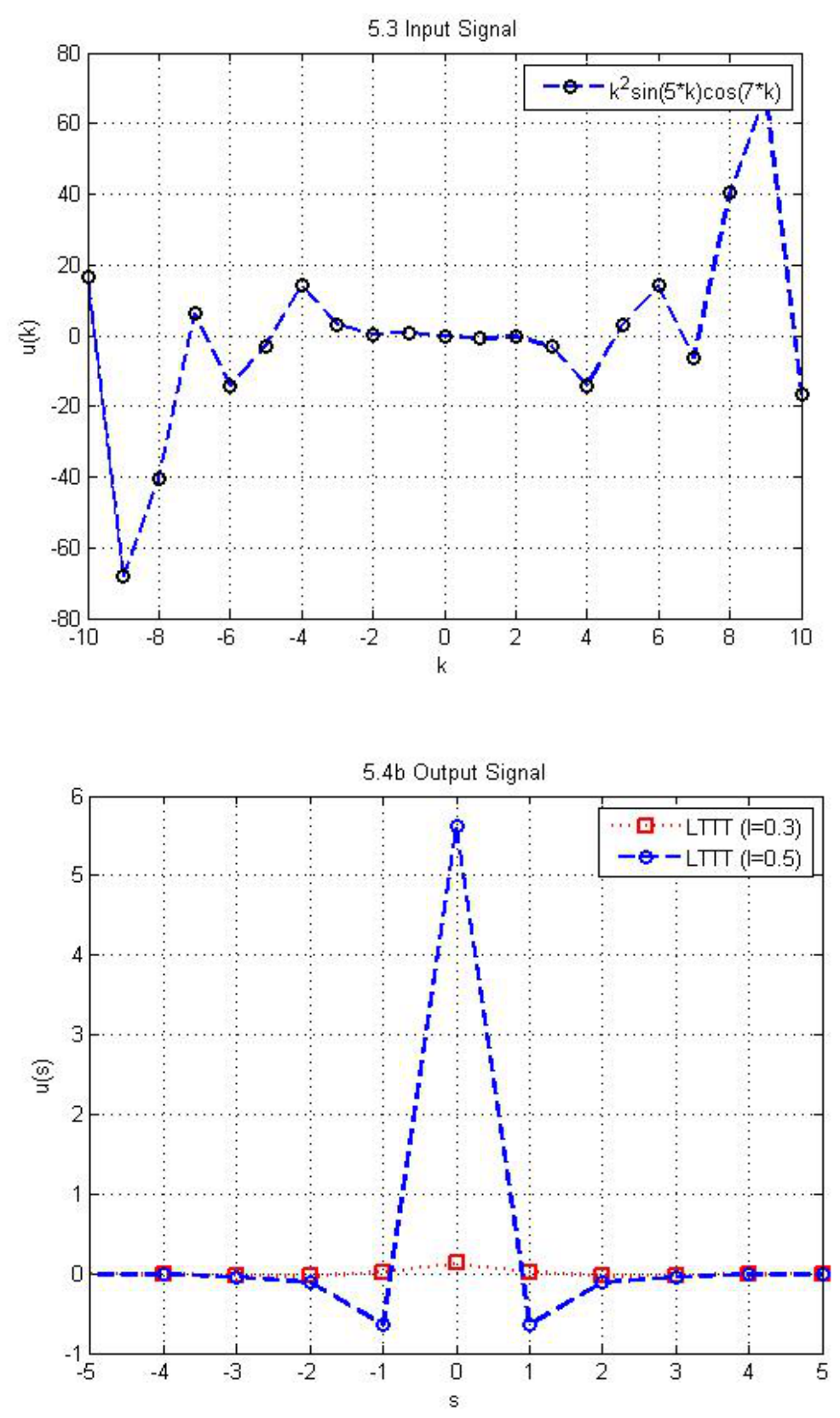

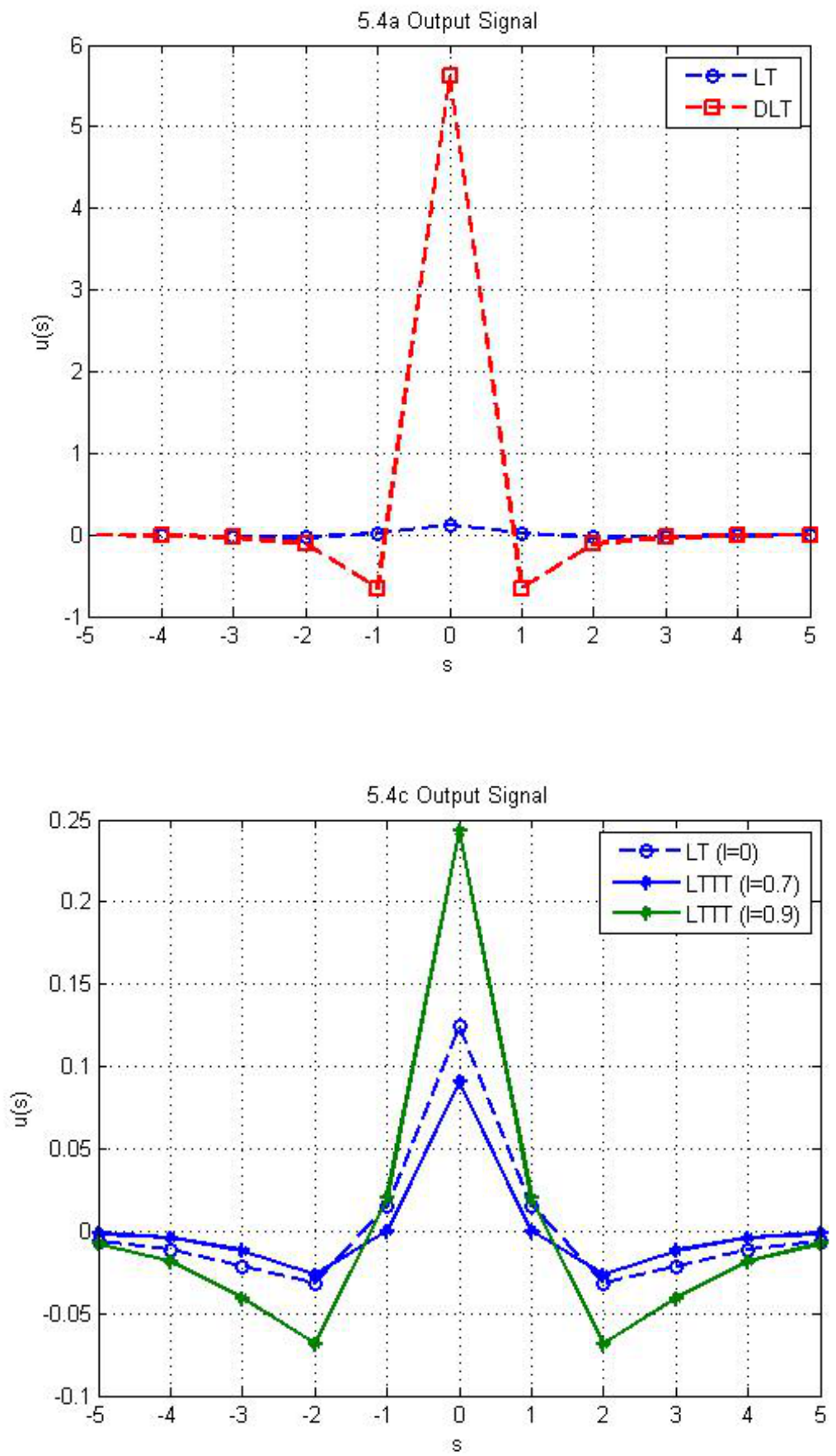

Conclusion: From the outcome of our findings, we observe with the help of the diagrams generated by MATLAB that LTTT gives innumerable outcomes by varying the Time Tuning Factor $\ell$ for the given input signal and this enables us to make a choice for an optimal one in DSP.

Acknowledgement: Catalyzed and financially supported by Tamilnadu State Council for Science and Technology, Dept. of Higher Education, Government of Tamilnadu. 


\section{References}

[1] Agarwal R.P., Difference Equations and Inequalities, Marcel Dekker, New York, 2000.

[2] A. N. Akansu and Poluri, " Walsh-like Nonlinear Phase Orthogonal Codes for Direct Sequences CDMA Communications", IEEE Trans. on Signal Processing, pp 3800-3806, July 2007.

[3] Aleksandar Ivic, Some Applications of Laplace Transforms in Analytic Number Theory, Novi Sad J. Math. Vol. 45, No. 1, 2015, 31-44.

[4] Bilal Atfeh, Laurent Baratchart, Juliette Leblond, Jonathan R. Partington, Bounded Extremal and Cauchy- Laplace Problems on the Sphere and Shell, J Fourier Anal Appl (2010)16:177-203.

[5] V. Britanak and K. R. Rao, " The Fast Generalized discrete Fourier Transforms: A unified Approach to the Discrete Sinusoidal Transforms Computation,” Siganal Processing, vol. 79, pp. 135-150, Dec 1999.

[6] G.B.A. Xavier, V.Chandrasekar, S.U. Vasanthakumar, and B. Govindan,Discrete Gamma(Factorial) Function and its Terms of a Generalized Difference Operator, Advances in Numerical Analysis, vol. 2012, Article ID 780646, 13 pages,2012.doi:10.1155/2012/780646

[7] G.B.A. Xavier, S.Sathya, S. U. Vasanthakumar, m-Series of the Generalized Difference Equation to Circular Functions, Int. Jour. of Mathematical Archive-4(7), (2013), 200-209.

[8] G.Britto Antony Xavier, B.Govindan, S.John Borg and M.Meganathan, Generalized Laplace Transform arrived from an Inverse Difference Operator, Global Journal of Pure and Applied Mathematics (accepted), 2016.

[9] Ivic A., The Laplace transform of the fourth moment of of the zeta-function, Univ. Beograd. Publ. Elektrotehn. Fak. Ser. Mat. 11 (2000), 41-48.

[10] Jutila, M., The Mellin transform of the square of Riemann’s zeta-function, Pe-riodica Math. Hung. 42 (2001), 179-190.

[11] M.M.S. Manuel, G.B.A. Xavier and E.Thandapani, Theory of Generalized Difference Operator and Its Applications, Far East Journal of Mathematical Sciences, 20(2) (2006), 163 - 171.

[12] M.M.S. Manuel and G.B.A. Xavier, Theory and Application of the Generalized Difference Operator of the $n^{\text {th }}$ Kind (Part - I), Demonstratio Mathematica, 45(1) (2012), 95 - 106.

[13] K.S.Miller, B.Ross , Fractional difference calculus, in "Univalent functions, fractional calculus, and the applications (Koriyama, 1988)", 139-152, Horwood, Chichester, 1989.

[14] Sedletskii, A.M., Fourier Transforms and Approximations, Gordon and Breach Science Publishers, Amsterdam 2000.

[15] Steven W. Smith, "The Scientist and Engineer’s Guide to Digital Signal Processing", Second Edition, California Technical Publishing San Diego, California, 1999.

[16] Wolfgang Erb., Optimally Space Localized Polynomials with Applications in Signal Processing, J Fourier Anal Appl (2012) 18 : pp 45 66 .

\section{AUTHOR PROFILE}

G. Britto Antony Xavier is currently working as an Associate Professor in the PG and Research Department of Mathematics, Sacred Heart College(Autonomous), Tirupattur. He has 24 years of teaching experience. He holds B.Sc(1980) and M.Sc(1990) from Madurai Kamaraj University, M.Phil (1991) from Manonmanium Sundaranar University. He has 110 publications in reputed in National and International Journals. He has published 3 books with ISSN numbers. His field of research interest includes Difference Equations, Partial Difference Equations and Fractional Calculus. He is also editor-in-chief of Journal of Computational Mathematica. He is also Life member of Indian Mathematical Society, Academy of Discrete Mathematics and Applications. He has visited 3 countries namely Portugal, Italy and France.

S. U. Vasantha Kumar is currently working as an Assistant Professor in the PG and Research Department of Mathematics, Sacred Heart College(Autonomous), Tirupattur. He has 7 years of teaching experience. He has completed B.Sc, M.Sc and M.Phil in Sacred Heart College from 2003 to 2009. He has 12 publications in reputed in National and International Journals. He has published 3 books with ISSN numbers. His field of research interest includes Difference Equations and Graph Theory.

M. Meganathan is currently research scholar in the PG and Research Department of Mathematics, Sacred Heart College(Autonomous), Tirupattur. He has 2 years of teaching experience. He has completed B.Sc, M.Sc and M.Phil in Sacred Heart College from 2010 to 2016. He has 13 publications in reputed in National and International Journals. His field of research interest includes Difference Equations and Fractional Calculus. 